\title{
BARROW'S INEQUALITY AND SIGNED ANGLE BISECTORS
}

\section{Branko Malešević And Maja Petrović}

Abstract. In this paper we give one extension of BARROW's type inequality in the plane of the triangle $\triangle A B C$ introduce signed angle bisectors.

Mathematics subject classification (2010): 51M16, 51M04, 14H50.

Keywords and phrases: Signed distance, BARROw's inequality.

\section{REFERENCES}

[1] G. W. I. S. AmARASINGHe, On the standard lengths of Angle Bisectors and Angle Bisector Theorem, Global Journal of Advanced Research on Classical and Modern Geometries, 1, 1 (2012), 15-27.

[2] D. F. BArrow, L. J. Mordell, Solution of Problem 3740, Amer. Math. Monthly, 44, (1937), 252 254.

[3] N. Dergiades, Signed distances and the Erdös-Mordell inequality, Forum Geom., 4, (2004), 67-68.

[4] P. ERdös, Problem 3740, Amer. Math. Monthly, 42, (1935), 396.

[5] R. FARouki, Pythagorean-Hodograph Curves: Algebra and Geometry Inseparable, Series: Geometry and Computing, Vol. 1, Springer 2008.

[6] W.-D. JiAng, An Inequality Involving the Angle Bisectors and an Interior Point of a Triangle, Forum Geometricorum, 8 (2008) 73-76.

[7] W.-D. JIANG AND M. BENCZE, Some geometric inequalities involving angle bisectors and medians of a triangle, Journal of Mathematical Inequalities, 5, 3 (2011), 363-369.

[8] H. LeE, Topics in Inequalities - Theorems and Techniques, Korea Institute for Advanced Study, Seoul, (http://www.normalesup.org/ kortchem/olympiades/Cours/Inegalites/tin2006.pdf)

[9] Z. LU, Erdös-Mordell inequality and beyond, Educational Talk at the UCI Math Club (28.11.2007), (http://www.math.uci.edu/ zlu/talks/2007-uci-mathclub/ucimathclub.pdf)

[10] Z. LU, Erdös-Mordell-type inequalities, Elemente der Mathematik, 63, (2008), 23-24.

[11] B. Malešević, M. Petrović, M. Obradović and B. Popkostantinović, On the Extension of the Erdös-Mordell Type Inequalities, Math. Inequal. Appl. 17, 1 (2014), 269-281.

[12] V. PAmbuccian, The Erdös-Mordell inequality is equivalent to non-positive curvature, Journal of Geometry, 88, (2008), 134-139.

[13] M. E. Saul, Hadamard's Plane Geometry: A Reader's Companion, American Mathematical Soc., 2010.

[14] P. YIU, Conic Construction of a Triangle from the Feet of Its Angle Bisectors, Journal of Geometry and Graphics, 12, 2 (2008), 172-182. (http://math.fau.edu/yiu/YiuFromBisectors .pdf)

[15] SH-H. WU AND ZH-H. ZHANG, A class of inequalities related to the angle bisectors and the sides of a triangle, J. Inequal. Pure Appl. Math., 7, 3 (2006), Article 108. 